\title{
Research on the Effect of Public R\&D Subsidies on R\&D Investment Expenditure of Technological Innovation Enterprises
}

\author{
Ailing Li \\ Harbin University of Commerce, Harbin 150028, China \\ ailing_li@qq.com
}

Keywords: Public R\&D Subsidies, R\&D Investment, Extrusion Effect, Stimulation Effect.

\begin{abstract}
As the product attribute of R\&D investment, enterprises are lack of the enthusiasm of R\&D investment. Public $R \& D$ subsidies may reduce the cost of $R \& D$ investment, and promote $R \& D$ investment of enterprises. However public $R \& D$ also has the extrusive effect to the $R \& D$ investment of enterprises. This paper presents evidence on the effects of public R\&D subsidies on the R\&D investment of technological innovation enterprises. The results show that public R\&D subsidies can stimulate the $R \& D$ investment of enterprises. There also exist different effects of public R\&D subsidies between different scales, proportion of state-owned shares and managerial ownership of enterprises.
\end{abstract}

\section{Introduction}

The R\&D investment of technological innovation enterprises is characterized by high input, high risk and long term, and its achievements have public goods quality. If only with voluntary R\&D investment of technological innovation enterprises, the level of R\&D investment will below the best R\&D investment level, so as to cause the market failure.

The governments have taken a positive measures to improve the R\&D investment activities of enterprises by public R\&D subsidies, tax incentive, subsidized interest. Although the aims of public $R \& D$ subsidies are to improve the spontaneous $R \& D$ investment of enterprises, the effects of public R\&D subsidies can be complete opposite: stimulation effect and extrusion effect. If public R\&D subsidies increase the enterprises' $R \& D$ investment, then stimulation effect works, otherwise extrusion effect works.(Levin, 1984; Wallsten, 2000).

\section{Hypothesis}

The investment of enterprise depends on expected return, risk and financial constraints in the market without government intervention. Therefore enterprises have no incentive to develop investment project with high risk and long period, especially in the case of the enterprises can not monopolize the proceeds of the investments. In fact R\&D investment falls into this category. In order to improve $R \& D$ investment level, public $R \& D$ subsidies are needed to reduce the cost and risk of R\&D investment and improve its expected return(Guellec etc. 2003; Hu, 1998).

Hypothesis 1a, public R\&D subsidies will stimulate the technological innovation enterprises to invest in $R \& D$ projects.

On the other hand, the activities of technological innovation enterprises' R\&D investment are differences in properties. Some kind of $R \& D$ investments is low risk with relatively high returns, some kind of R\&D investment is important to the enterprises development, consequently enterprises will do it anyway. Therefore if public R\&D subsidies are put on these projects, that will decrease the level of enterprises spontaneous R\&D investments(Lichtenberg, 1987).

Hypothesis 1b: public R\&D subsidies will crowd out the R\&D investment of technological innovation enterprises.

The effect of public R\&D subsidies on different kinds of technological innovation enterprises can be different, such as scale, proportion of state-owned shares,agency problem and so on. 
Hypothesis 2a: the effect of public R\&D subsidies on technological innovation enterprises R\&D investment decrease with the enterprises scale increase.

Hypothesis 2b: the effect of public R\&D subsidies on technological innovation enterprises R\&D investment negatively correlate withproportion of state-owned shares of the enterprises.

Hypothesis 2c: the effect of public R\&D subsidies on technological innovation enterprises R\&D investment decrease with the agency problem of the enterprises increase.

\section{The Model and the Variables}

We assume the following equations to describe the effection of public R\&D subsidies on enterprises R\&D investment:

$$
\begin{aligned}
R \& D_{i t}= & f\left(G_{\text {OOV }}, \text { size }_{i t}, \mathrm{CF}_{i t}, \text { Lev }_{i t}, \text { Executive }_{i t}, \text { Roa }_{i t}, \text { eps }_{i t}, \text {,rowth }_{i t}\right) \\
& +\sum \text { Year }+\sum \text { Ind }+\varepsilon_{i t} \\
R \& D_{i t}= & f\left(\text { gov_dum }_{i t}, \text {,ize }_{i t}, \mathrm{CF}_{i t}, \text { Lev }_{i t}, \text { Executive }_{i t}, \text { Roa }_{i t}, \text {,ps }_{i t}, \text { growth }_{i t}\right) \\
& +\sum \text { year }+\sum \text { Ind }+\varepsilon_{i t}
\end{aligned}
$$

$G O V_{i t}$ or gov_dum $i t$ is used as a proxy for the effect of public R\&D subsidies, so that hypothesis 1a would suggest a positive and significant coefficient. Otherwise hypothesis $1 \mathrm{~b}$ would be proved.

The describtion of variables are as follows:

1.The explained variable:

$R \& D_{i t}$ is the R\&D investment of listed technological innovation companies which is disclosed by the annual report. The information of $\mathrm{R} \& \mathrm{D}$ investment is reported in the main business of the annual report of listed companies. Because of the lack of data before 2010 annual report, we find the R\&D investment in "administration expense" or "the cash flow related with business activities".

2.The explanatory variables: $G O V_{i t}$ is the natural logarithm of the public R\&D subsidies, which is the sum of $R \& D$ project subsidies, new products subsidies, innovation awards and so on.In order to avoid the sample selection bias, in model (2) the public R\&D subsidies variable gov_dum ${ }_{i t}$ is a dichotomous variable that takes the value of 1 if there is public R\&D subsidies, and a null value if not.

3. The control variables: enterprise scale ( size $_{i t}$ ) is the natural logrithm of total asset, cash flow ratio $\left(C F_{i t}\right)$ is the ratio of "operating cash flow" to "fixed asset", debt ratio ( $\left.L e v_{i t}\right)$ is the ratio of total debt to total asset, executives share $\left(\right.$ Executive $\left._{i t}\right)$ is a dummy variable, growth rate $\left(\right.$ growth $\left._{i t}\right)$ is the main business revenue growth.

\section{Descriptive Statistics}

Table 1 shows descriptive statistics for observations. In order to reduce the influence of outliers, we delete the data of each variables which is out of range $1 \%$ and $99 \%$. Table 2 shows descriptive statistics in each year.

Table 1. Descriptive Statistics

\begin{tabular}{c|c|c|c|c|c}
\hline Variables & Median & Mean & Std Dev & Minimum & Maximum \\
\hline$R \& D_{i t}$ & 17.42286 & 17.30836 & 1.736954 & 8.337466 & 22.90133 \\
\hline$G O V_{i t}$ & 14.4033 & 14.36531 & 1.863658 & 7.090077 & 21.48748 \\
\hline size $_{i t}$ & 21.53529 & 21.60844 & 1.239726 & 10.8422 & 26.64656 \\
\hline$C F_{i t}$ & 0.1945464 & 0.2039045 & 2.677834 & -123.8242 & 14.78063 \\
\hline Lev $_{i t}$ & 0.4589497 & 0.6620081 & 4.392573 & 0.0290972 & 142.7178 \\
\hline Executive $_{i t}$ & 0 & 0.0242041 & 0.0771209 & 0 & 0.6921961 \\
\hline Roa $_{i t}$ & 0.0412595 & 0.0322985 & 1.344178 & -64.81915 & 20.78764 \\
\hline eps $_{i t}$ & 0.23 & 0.3485072 & 0.5364539 & -2.72 & 6.28 \\
\hline growth $_{i t}$ & 0.115937 & 0.3642874 & 2.372684 & -1.605846 & 62.18187 \\
\hline & & & & &
\end{tabular}


Table 2. Descriptive Statistics by Year

\begin{tabular}{c|c|c|c|c|c|c}
\hline year & \multicolumn{3}{|c|}{ samples } & \multicolumn{3}{c}{ Samples with R\&D } \\
\hline & $\begin{array}{c}\text { Firms } \\
\text { Observations }\end{array}$ & R\&D & Ratio & $\begin{array}{c}\text { Firms } \\
\text { Observations }\end{array}$ & $\begin{array}{c}\text { Public } \\
\text { R\&D } \\
\text { Subsidies }\end{array}$ & Ratio \\
\hline 2008 & 450 & 210 & 0.466667 & 210 & 134 & 0.638095 \\
\hline 2009 & 451 & 359 & 0.796009 & 359 & 258 & 0.718663 \\
\hline 2010 & 451 & 379 & 0.840355 & 379 & 283 & 0.746702 \\
\hline 2011 & 451 & 422 & 0.935698 & 422 & 326 & 0.772512 \\
\hline 2012 & 451 & 437 & 0.968958 & 437 & 358 & 0.819222 \\
\hline 2013 & 449 & 431 & 0.959911 & 431 & 357 & 0.828306 \\
\hline
\end{tabular}

\section{Regression Results}

\subsection{Full Sample Results}

The $\chi^{2}$ results of hausman test on model (1) and model (2) are 19.94 and 19.49, so we ues the random-effect model to regress. Table 3 shows that the results of the effect of public R\&D subsidies on technological innovation enterprises R\&D investments.

Table 3. Public R\&D Subsidies Regression

\begin{tabular}{|c|c|c|c|c|}
\hline & \multicolumn{2}{|c|}{ Model(1) } & \multicolumn{2}{|c|}{ Model(2) } \\
\hline & $R \& D_{i t}$ & $R \& D_{i t+1}$ & $R \& D_{i t}$ & $R \& D_{i t+1}$ \\
\hline$G O V_{i t}$ & $\begin{array}{c}0.15 * * * \\
(8.62)\end{array}$ & $\begin{array}{l}0.03^{*} \\
(1.90)\end{array}$ & & \\
\hline gov_dum $_{i t}$ & & & $\begin{array}{c}2.09 * * * \\
(8.33)\end{array}$ & $\begin{array}{c}0.433^{*} \\
(1.82)\end{array}$ \\
\hline size $_{i t}$ & $\begin{array}{l}0.33 * * \\
(2.24)\end{array}$ & $\begin{array}{c}0.54 * * * \\
(3.72)\end{array}$ & $\begin{array}{c}0.41 * * * \\
(2.77)\end{array}$ & $\begin{array}{c}0.56 * * * \\
(3.83)\end{array}$ \\
\hline$C F_{i t}$ & $\begin{array}{c}0.02 \\
(0.23) \\
\end{array}$ & $\begin{array}{c}0.16^{*} \\
(1.88) \\
\end{array}$ & $\begin{array}{c}0.02 \\
(0.23) \\
\end{array}$ & $\begin{array}{c}0.16^{*} \\
(1.87) \\
\end{array}$ \\
\hline$L e v_{i t}$ & $\begin{array}{l}-0.12^{*} \\
(-1.90)\end{array}$ & $\begin{array}{c}-0.16^{* *} \\
(-2.31)\end{array}$ & $\begin{array}{c}-0.11^{*} \\
(-1.81)\end{array}$ & $\begin{array}{l}-016 * * \\
(-2.29)\end{array}$ \\
\hline Executive $_{i t}$ & $\begin{array}{c}6.29 * * * \\
(3.64)\end{array}$ & $\begin{array}{c}5.38^{* * * *} \\
(3.12)\end{array}$ & $\begin{array}{c}6.34 * * * \\
(3.66)\end{array}$ & $\begin{array}{c}5.39 * * * \\
(3.12)\end{array}$ \\
\hline $\operatorname{Roa}_{i t}$ & $\begin{array}{c}-0.72 \\
(-0.78) \\
\end{array}$ & $\begin{array}{c}-0.32 \\
(-0.26) \\
\end{array}$ & $\begin{array}{c}-0.71 \\
(-0.77)\end{array}$ & $\begin{array}{c}-0.31 \\
(-0.25)\end{array}$ \\
\hline $\mathrm{eps}_{i t}$ & $\begin{array}{c}0.86^{* * * *} \\
(3.04)\end{array}$ & $\begin{array}{l}0.60 * * \\
(2.10)\end{array}$ & $\begin{array}{c}0.87 * * * \\
(3.09)\end{array}$ & $\begin{array}{l}0.60 * * \\
(2.10)\end{array}$ \\
\hline growth $_{i t}$ & $\begin{array}{c}0.00 \\
(0.03) \\
\end{array}$ & $\begin{array}{c}-0.02 \\
(-0.46) \\
\end{array}$ & $\begin{array}{c}0.000 \\
0(0.02) \\
\end{array}$ & $\begin{array}{c}-0.02 \\
(-0.47) \\
\end{array}$ \\
\hline Constant & $\begin{array}{c}-0.89 \\
(-0.28) \\
\end{array}$ & $\begin{array}{c}1.20 \\
(0.39) \\
\end{array}$ & $\begin{array}{c}-2.51 \\
(-0.81) \\
\end{array}$ & $\begin{array}{c}0.87 \\
0(0.28) \\
\end{array}$ \\
\hline Ind/year & Contral & Contral & Contral & Contral \\
\hline R2-within & 0.3966 & 0.1737 & 0.3961 & 0.1742 \\
\hline R2-between & 0.1787 & 0.1590 & 0.1734 & 0.1563 \\
\hline Wald chi 2 & $1520.61 * * *$ & 435.64 & $1513.95^{* * *}$ & $435.49 * * *$ \\
\hline $\mathrm{N}$ & 2645 & 2195 & 2645 & 2195 \\
\hline
\end{tabular}

$* * * \mathrm{p}<0.01 ; * * \mathrm{p}<0.05 ; * \mathrm{p}<0.1 ; \mathrm{z}$ values in parentheses 
Table 3 shows the result of our investigations into the impact of public R\&D subsidies on technological innovation enterprises. The list 2 and 4 are the regressions of model (1) and (2) with $R \& D_{i t}$, and the list 3 and 5 are the regressions with $R \& D_{i t+1}$, which is the lag of R\&D investment.

Table 3 shows that the coefficient of $G O V_{i t}$ and gov_dum it $_{\text {are }}$ positive and significant at the $1 \%$ significance level, hypothesis 1a is proved. The financial market of China is developing, where the innovation can not be fully protected. So that the technological innovation enterprises don't have much enthusiasm to invest in $R \& D$ project. Public R\&D subsidies which can reduce the cost and risk of R\&D investment will improve the level of voluntary R\&D investment efficiently.

\subsection{Grouped Samples Results}

(1) Scale

To test hypothesis $2 \mathrm{a}$, we regress the effect of public R\&D subsidies on different scale of enterprises which is grouped by median. Table 4 shows the results.

Table 4. Public R\&D Subsidies Regression by Scale

\begin{tabular}{|c|c|c|c|c|}
\hline & \multicolumn{2}{|c|}{ Large-scale } & \multicolumn{2}{|c|}{ Small-scale } \\
\hline & Model(1) & $\operatorname{Model}(2)$ & Model(1) & Model(2) \\
\hline$G O V_{i t}$ & $\begin{array}{c}0.10^{* * * *} \\
(4.15)\end{array}$ & & $\begin{array}{c}0.19 * * * \\
(7.79)\end{array}$ & \\
\hline gov_dum $_{i t}$ & & $\begin{array}{c}1.44^{* * * *} \\
(3.81)\end{array}$ & & $\begin{array}{c}2.48 * * * \\
(7.57)\end{array}$ \\
\hline $\operatorname{size}_{i t}$ & $\begin{array}{l}0.42 * \\
(1.76) \\
\end{array}$ & $\begin{array}{c}0.48 * * \\
(1.99) \\
\end{array}$ & $\begin{array}{l}0.31 * \\
(1.71) \\
\end{array}$ & $\begin{array}{c}0.38 * * \\
(1.93)\end{array}$ \\
\hline$C F_{i t}$ & $\begin{array}{c}-0.23 \\
(-1.16) \\
\end{array}$ & $\begin{array}{c}-0.23 \\
(-1.18) \\
\end{array}$ & $\begin{array}{c}-0.02 \\
(-0.23) \\
\end{array}$ & $\begin{array}{c}-0.02 \\
(-0.21) \\
\end{array}$ \\
\hline$L e v_{i t}$ & $\begin{array}{c}0.46 \\
(0.38)\end{array}$ & $\begin{array}{c}0.47 \\
(0.38)\end{array}$ & $\begin{array}{l}-0.14^{*} \\
(-1.90)\end{array}$ & $\begin{array}{l}-0.14^{*} \\
(-1.84)\end{array}$ \\
\hline Executive $_{i t}$ & $\begin{array}{l}5.30 * * \\
(2.56) \\
\end{array}$ & $\begin{array}{l}5.31 * * \\
(2.51) \\
\end{array}$ & $\begin{array}{l}4.57 * * \\
(2.30)\end{array}$ & $\begin{array}{l}4.68 * * \\
(2.36)\end{array}$ \\
\hline $\operatorname{Roa}_{i t}$ & $\begin{array}{c}2.34 \\
(0.49) \\
\end{array}$ & $\begin{array}{c}2.63 \\
(0.56) \\
\end{array}$ & $\begin{array}{c}-1.52 \\
(-1.48) \\
\end{array}$ & $\begin{array}{c}-1.50 \\
(-1.46) \\
\end{array}$ \\
\hline $\mathrm{eps}_{i t}$ & $\begin{array}{c}0.41 * * \\
(1.96) \\
\end{array}$ & $\begin{array}{c}0.41 * * \\
(1.94) \\
\end{array}$ & $\begin{array}{c}2.15 * * * \\
(3.81)\end{array}$ & $\begin{array}{c}2.18 * * * \\
(3.85)\end{array}$ \\
\hline growth $_{i t}$ & $\begin{array}{c}0.01 \\
(0.15) \\
\end{array}$ & $\begin{array}{c}0.01 \\
(0.11) \\
\end{array}$ & $\begin{array}{c}0.02 \\
(0.37) \\
\end{array}$ & $\begin{array}{c}0.03 \\
(0.44) \\
\end{array}$ \\
\hline Constant & $\begin{array}{c}-3.40 \\
(-0.66) \\
\end{array}$ & $\begin{array}{c}-4.66 \\
(-0.90) \\
\end{array}$ & $\begin{array}{c}6.73 \\
(0.91) \\
\end{array}$ & $\begin{array}{c}4.90 \\
(0.66) \\
\end{array}$ \\
\hline Ind & Control & Control & Control & Control \\
\hline year & Control & Control & Control & Control \\
\hline R2-within & 0.3705 & 0.3696 & 0.3943 & 0.3934 \\
\hline R2-between & 0.2451 & 0.2419 & 0.2502 & 0.2470 \\
\hline Wald chi2 & $642.97 * * *$ & $639.36 * * *$ & $755.15 * * *$ & 750.16 *** \\
\hline $\mathrm{N}$ & 1337 & 1337 & 1308 & 1308 \\
\hline
\end{tabular}

$* * * \mathrm{p}<0.01 ; * * \mathrm{p}<0.05 ; * \mathrm{p}<0.1 ; \mathrm{z}$ values in parentheses

Table 4 shows there exist significant differences between large-scale group and small-scale group. The public R\&D subsidies have more obvious influence on small technological innovation enterprises than large ones, hypothesis $2 \mathrm{a}$ is proved. 


\section{(2) State-Owned Shares}

To test hypothesis $2 b$, we regress the effect of public $R \& D$ subsidies on different proportions of state-owned shares which is grouped by median. Table 5 shows the results.

Table 5. Public R\&D Subsidies Regression by State-Owned Shares

\begin{tabular}{|c|c|c|c|c|}
\hline & \multicolumn{2}{|c|}{ High } & \multicolumn{2}{|c|}{ Low } \\
\hline & Model(1) & Model(2) & Model(1) & Model(2) \\
\hline$G O V_{i t}$ & $\begin{array}{c}0.12 * * * \\
(3.61)\end{array}$ & & $\begin{array}{c}0.15^{* * * *} \\
(7.55)\end{array}$ & \\
\hline gov_dum $_{i t}$ & & $\begin{array}{c}1.63 \\
(3.38)\end{array}$ & & $\begin{array}{c}2.14 * * * \\
(7.30)\end{array}$ \\
\hline $\operatorname{size}_{i t}$ & $\begin{array}{l}0.44^{*} \\
(1.65)\end{array}$ & $\begin{array}{l}0.50^{*} \\
(1.87)\end{array}$ & $\begin{array}{c}0.34 * * \\
(2.07)\end{array}$ & $\begin{array}{c}0.42 * * \\
(2.52)\end{array}$ \\
\hline$C F_{i t}$ & $\begin{array}{c}-0.25 \\
(-0.75)\end{array}$ & $\begin{array}{c}-0.27 \\
(-0.80)\end{array}$ & $\begin{array}{c}0.01 \\
(0.09)\end{array}$ & $\begin{array}{c}0.01 \\
(0.12)\end{array}$ \\
\hline$L e v_{i t}$ & $\begin{array}{c}-0.92 * * \\
(-2.36)\end{array}$ & $\begin{array}{c}-0.91 * * \\
(-2.34)\end{array}$ & $\begin{array}{l}-0.12^{*} \\
(-1.87)\end{array}$ & $\begin{array}{l}-0.12 * \\
(-1.78)\end{array}$ \\
\hline Executive $_{i t}$ & $\begin{array}{l}7.66^{*} \\
(1.68)\end{array}$ & $\begin{array}{l}7.54 * \\
(1.79)\end{array}$ & $\begin{array}{c}6.28 * * * \\
(3.66)\end{array}$ & $\begin{array}{c}6.34 * * * \\
(3.68)\end{array}$ \\
\hline $\operatorname{Roa}_{i t}$ & $\begin{array}{c}1.91 \\
(0.97)\end{array}$ & $\begin{array}{c}1.91 \\
(0.96)\end{array}$ & $\begin{array}{c}-0.98 \\
(-0.92)\end{array}$ & $\begin{array}{c}-0.94 \\
(-0.88)\end{array}$ \\
\hline $\mathrm{eps}_{i t}$ & $\begin{array}{c}0.51 \\
(0.97)\end{array}$ & $\begin{array}{c}0.51 \\
(0.97)\end{array}$ & $\begin{array}{c}1.05 * * * \\
(3.12)\end{array}$ & $\begin{array}{c}1.08 * * * \\
(3.21)\end{array}$ \\
\hline growth $_{i t}$ & $\begin{array}{c}-0.02 \\
(-0.30)\end{array}$ & $\begin{array}{c}-0.02 \\
(-0.34)\end{array}$ & $\begin{array}{c}0.00 \\
(0.07)\end{array}$ & $\begin{array}{c}0.00 \\
(0.10)\end{array}$ \\
\hline Constant & $\begin{array}{c}-3.10 \\
(-0.55) \\
\end{array}$ & $\begin{array}{c}-4.30 \\
(-0.76) \\
\end{array}$ & $\begin{array}{c}-0.49 \\
(-0.14) \\
\end{array}$ & $\begin{array}{c}-2.07 \\
(-0.59) \\
\end{array}$ \\
\hline Ind/year & Control & Control & Control & Control \\
\hline R2-within & 0.4401 & 0.4394 & 0.3125 & 0.3124 \\
\hline R2-between & 0.4083 & 0.4064 & 0.2344 & 0.2285 \\
\hline Wald chi2 & $555.22 * * *$ & $552.60 * * *$ & $756.66 * * *$ & $752.43 * * *$ \\
\hline $\mathrm{N}$ & 785 & 785 & 1860 & 1860 \\
\hline
\end{tabular}

$* * * \mathrm{p}<0.01 ; * * \mathrm{p}<0.05 ; * \mathrm{p}<0.1 ; \mathrm{z}$ values in parentheses

Table 5 shows there exist significant differences between high state-owned shares group and low state-owned shares group. Hypothesis $2 \mathrm{~b}$ is proved.

\section{(3) Management Ownership}

To test hypothesis $2 c$, we regress the effect of public R\&D subsidies on different scale of management ownership which is grouped by median. Table 6 shows the results. 
Table 6. Public R\&D Subsidies Regression by Management Ownership

\begin{tabular}{|c|c|c|c|c|}
\hline & \multicolumn{2}{|c|}{ High management ownership } & \multicolumn{2}{|c|}{ Low management ownership } \\
\hline & Model(1) & $\operatorname{Model}(2)$ & Model(1) & Model(2) \\
\hline$G O V_{i t}$ & $\begin{array}{c}0.12^{* * * *} \\
(4.68)\end{array}$ & & $\begin{array}{c}0.16^{* * *} \\
(6.72)\end{array}$ & \\
\hline gov_dum $_{i t}$ & & $\begin{array}{c}1.61^{* * * *} \\
(4.32)\end{array}$ & & $\begin{array}{l}2.26^{* * * *} \\
(6.68)\end{array}$ \\
\hline $\operatorname{size}_{i t}$ & $\begin{array}{c}0.14 \\
(0.65) \\
\end{array}$ & $\begin{array}{c}0.21 \\
(0.93) \\
\end{array}$ & $\begin{array}{c}0.67^{* * * *} \\
(3.49)\end{array}$ & $\begin{array}{c}0.75^{* * * *} \\
(3.94)\end{array}$ \\
\hline$C F_{i t}$ & $\begin{array}{c}-0.14 \\
(-0.59)\end{array}$ & $\begin{array}{c}-0.14 \\
(-0.59)\end{array}$ & $\begin{array}{c}-0.04 \\
(-0.50)\end{array}$ & $\begin{array}{c}-0.04 \\
(-0.54)\end{array}$ \\
\hline$L e v_{i t}$ & $\begin{array}{c}-0.26 \\
(-0.52)\end{array}$ & $\begin{array}{c}-0.24 \\
(-0.47)\end{array}$ & $\begin{array}{l}-0.11^{*} \\
(-1.65)\end{array}$ & $\begin{array}{c}-0.10 \\
(-1.57)\end{array}$ \\
\hline Executive $_{i t}$ & $\begin{array}{l}4.74^{* * * *} \\
(2.66)\end{array}$ & $\begin{array}{l}4.81^{* * * *} \\
(2.69)\end{array}$ & $\begin{array}{c}-3.15 \\
(-0.15)\end{array}$ & $\begin{array}{c}-3.88 \\
(-0.18)\end{array}$ \\
\hline $\operatorname{Roa}_{i t}$ & $\begin{array}{c}12.63^{* * * *} \\
(2.97)\end{array}$ & $\begin{array}{c}13.01^{* * * *} \\
(3.06)\end{array}$ & $\begin{array}{l}-1.35 \\
(-1.38)\end{array}$ & $\begin{array}{c}-1.36 \\
(-1.39)\end{array}$ \\
\hline eps $_{i t}$ & $\begin{array}{c}-0.52 \\
(-0.88) \\
\end{array}$ & $\begin{array}{c}-0.53 \\
(0.89) \\
\end{array}$ & $\begin{array}{l}0.90^{* *} \\
(2.49)\end{array}$ & $\begin{array}{l}0.90^{* *} \\
(2.49)\end{array}$ \\
\hline growth $_{i t}$ & $\begin{array}{l}0.040 \\
(0.43) \\
\end{array}$ & $\begin{array}{c}0.04 \\
(0.37) \\
\end{array}$ & $\begin{array}{c}-0.01 \\
(-0.31) \\
\end{array}$ & $\begin{array}{c}-0.01 \\
(-0.28)\end{array}$ \\
\hline Constant & $\begin{array}{c}4.40 \\
(0.94) \\
\end{array}$ & $\begin{array}{c}3.10 \\
(0.66) \\
\end{array}$ & $\begin{array}{l}-9.55^{* *} \\
(-2.34) \\
\end{array}$ & $\begin{array}{c}-11.33^{* * * *} \\
(-2.78)\end{array}$ \\
\hline Ind/year & Control & Control & Control & Control \\
\hline $\mathrm{R}^{2}$-within & 0.3135 & 0.3118 & 0.4620 & 0.4627 \\
\hline $\mathrm{R}^{2}$-between & 0.3235 & 0.3241 & 0.2462 & 0.2406 \\
\hline Wald chi2 & $575.16^{* * *}$ & $570.49 * * *$ & $966.46 * * *$ & $967.14 * * *$ \\
\hline $\mathrm{N}$ & 1329 & 1329 & 1316 & 1316 \\
\hline
\end{tabular}

$* * * \mathrm{p}<0.01 ; * * \mathrm{p}<0.05 ; * \mathrm{p}<0.1 ; \mathrm{z}$ values in parentheses

Table 6 shows there exist significant differences between high management ownership group and low management ownership group. Hypothesis $2 \mathrm{c}$ is proved.

\section{Conclusion}

This paper studies the effect of public $R \& D$ subsidies on $R \& D$ investment of technological innovation enterprises. $R \& D$ investment is characterized by high risk and long period, so that technological innovation enterprises have no incentive to do it. The result shows that public R\&D subsidies can improve the level of $R \& D$ activities of technological innovation enterprises. It also shows that public R\&D subsidies have different effect on different kind of technological innovation enterprises

This work is supported by Philosophy and Social Sciences Youth Program of Heilongjiang Province (16JYC07).

\section{References}

[1]. Levin R, Reiss P. C., Tests of a Schumpeterian Model of R\&D and Market SructureR\&D, Patents, and Productivity [J]. University of Chicago Press, 1984: 175-208.

[2]. Wallsten S. J., The Effects of Government-Industry R\&D Programs on Private R\&D: the Case of the Small Business Innovation Research program [J]. RAND Journal of Economics, 2000, 31(1): 82-100. 
[3]. Guellec D., Van Pottelsberghe De La Potterie B. The Impact of Public R\&D Expenditure on Business R\&D [J]. Economics of innovation and new technology, 2003, 12(3): 225-243.

[4]. Hamberg D., R\&D [J]. Essays on the Economics of Research and Development, 1966.

[5]. Hu X., Schiantarelli F., Investment and Capital Market Imperfections: A Switching Regression Approach using Firm Panel Data [J]. The Review of Economics and Statistics, 1998, (53):466479.

[6]. Lichtenberg F. R., the Effect of the Composition of R\&D Spending [J]. Southern Journal of Economics, 1987,(4): 342-349.

[7]. Hall B. H., Oriani R., Does the Market Value R\&D Investment by European Firms? Evidence from a Panel of Manufacturing Firms in France, Germany, and Italy [J]. International Journal of Industrial Organization, 2006, 24(5): 971-993.

[8]. Ortega-Argilés R., Moreno R., Caralt J S., Ownership Structure and Innovation: Is there a Real Link? [J]. the Annals of Regional Science, 2005, 39(4): 637-662.

[9]. KaplanS., ZingalesL., Do investment-cash flow sensitivities provide useful measures of financing constraints?[J]. Quarterly Journal of Economics, 1997, (112):169-215.

[10]. WesterlundJ., Testing for Error Correction in Panel Data[J].Oxford Bulletin of Economics and Statistics ,2007,.(69): 709-748.

[11]. PersynD., J. Westerlund.,Error Correction Based Cointegration Tests for Panel Data[J]. Stata Journal, 2008, 8(2):232-241. 